\title{
SYSTEMIC LUPUS ERYTHEMATOSUS (SLE) COMPLICATED BY NEUROMYELITIS OPTICA: CASE SERIES FROM A TERTIARY PAEDIATRIC RHEUMATOLOGY CENTRE
}

\author{
D. Maritsi, M. Al-Obadi, S. Melo-Gomes, C. Edelsten, C.A. Pilkington \\ Rheumatology, Great Ormond Street Hospital for Children NHS Trust, London, UK
}

Objective: To identify the presence of Neuromyelitis optica (NMO) in juvenile SLE patients.

Background: NMO is a rare autoimmune demyelinating disease of the central nervous system, manifesting with transverse myelitis involving three or more continuous spinal segments and optic neuritis in the presence of NMO-IgG antibodies.

Methods: Retrospective study of SLE patients with CNS symptoms, diagnosed from 2000 to2010 and review of clinical data, laboratory and MRI findings.

Setting: A tertiary referral centre for juvenile SLE.

Results: A total of 210 SLE patients were indentified, 39 of which had manifestations of potential CNS involvement and underwent CNS imaging including the spinal cord. Three were indentified with probable NMO, which was confirmed in two $(0.9 \%)$. Both patients were adolescent females of Caucasian origin. In one patient NMO was the first manifestation of SLE. In the other NMO developed three years following diagnosis of SLE. They both presented with deterioration of visual acuity, localized spinal tenderness and malaise. NMO was confirmed based on MRI findings and the presence of raised Aquaporin-4-IgGantibodies. NMO had a relapsing course and interestingly NMO relapses coincided with SLE disease flareup, both of which responded to treatment simultaneously.

Discussion: SLE is a multisystemic autoimmune disease and $25 \%$ of patients develop CNS involvement. While NMO has been described in adult patients with SLE, these cases derive specifically from a paediatric population. We believe that SLE and NMO are parts of the same disease spectrum. When this condition is noticed in patients with refractory, long standing SLE, prognosis is guarded. 